\title{
Efficacy and Safety of Tocilizumab Treatment COVID- 19 Patients: A Case-Control Study and Meta-Analysis
}

\author{
Weijun Jiang · Weiwei Li · Qiuyue Wu - Ying Han · Jing Zhang • \\ Tao Luo $\cdot$ Yanju Guo $\cdot$ Yang Yang $\cdot$ Peiran Zhu $\cdot$ Xinyi Xia (B)
}

Received: March 20, 2021 / Accepted: June 15, 2021 / Published online: July 9, 2021

(C) The Author(s) 2021

\begin{abstract}
Introduction: As the pandemic progresses, the pathophysiology of COVID-19 is becoming more apparent, and the potential for tocilizumab is increasing. However, the clinical efficacy and safety of tocilizumab in the treatment of COVID-19 patients remain unclear.

Methods: To assess the efficacy and safety of tocilizumab treatment in COVID-19 patients, we performed a retrospective case-control study. The study was conducted, including 95 patients treated with tocilizumab plus standard treatment and matched controls with 95 patients treated with standard treatment therapy by propensity score from February to April
\end{abstract}

W. Jiang · W. Li · Q. Wu · Y. Han · J. Zhang ·

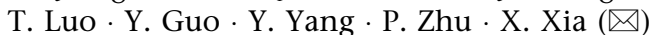

COVID-19 Research Center, Institute of Laboratory

Medicine, Jinling Hospital, Nanjing University

School of Medicine, The First School of Clinical

Medicine, Southern Medical University, Nanjing

210002, Jiangsu, China

e-mail: xinyixia@nju.edu.cn

X. Xia

Joint Expert Group for COVID-19, Department of Laboratory Medicine and Blood Transfusion, Wuhan Huoshenshan Hospital, Wuhan 430100, Hubei, China

X. Xia

Department of Laboratory Medicine and Blood

Transfusion, Wuhan Huoshenshan Hospital,

Wuhan 430100, Hubei, China
2020. We searched some databases using the search terms for studies published from January 1, 2020, to June 1, 2021.

Results: Our case-control study found a lower mortality rate in the tocilizumab treatment group than in the standard treatment group $(9.47 \%$ versus $16.84 \%, P=0.134)$, but the results were not statistically significant. We also found that the mortality rate in tocilizumab treatment groups was significantly lower than in the standard treatment group in the stratified ICU analysis (OR 0.52, 95\% CI 0.44-0.61, $P=0.048$ and OR $0.31,95 \%$ CI $0.10-0.99$, $P=0.044$ ). We selected 49 studies (including 6568 cases and 11,660 controls) that met the inclusion criteria in the meta-analysis. In the overall analysis, we performed a meta-analysis that showed significantly decreased mortality after patients received tocilizumab (OR 0.81, 95\% CI 0.69-0.95, $P=0.008)$. We also revealed significant associations within some subgroups. The sequential trial analysis showed a truepositive result. No significant associations were observed between tocilizumab and elevated secondary infection risk, discharge, adverse events, and mechanical ventilation in the overall analysis.

Conclusion: Tocilizumab significantly decreased mortality in COVID-19 patients with no increased discharge, secondary infection risk, adverse events, and mechanical ventilation in a meta-analysis. Our data suggest that clinicians should pay attention to tocilizumab 
therapy as an effective and safe treatment for COVID-19 patients.

Keywords: COVID-19; Efficacy; Meta-analysis; Safety; Tocilizumab

\section{Key Summary Points}

\section{Why carry out this study?}

As the pandemic progresses, the pathophysiology of COVID-19 is becoming more apparent, and the potential for tocilizumab is increasing.

However, the clinical efficacy and safety of tocilizumab in the treatment of COVID-19 patients remain unclear.

\section{What was learned from the study?}

The study was conducted, including 95 patients treated with tocilizumab plus standard treatment and matched controls with 95 patients treated with standard treatment therapy by propensity score from February to April 2020. We searched some databases using the search terms for studies published from January 1, 2020, to June 1, 2021.

Our case-control study found a lower mortality rate in the tocilizumab treatment group than in the standard treatment group $(9.47 \%$ versus $16.84 \%$, $P=0.134)$, but the results were not statistically significant.

We selected 49 studies (including 6568 cases and 11,660 controls) that met the inclusion criteria in the meta-analysis. In the overall analysis, we performed a metaanalysis that showed significantly decreased mortality after patients received tocilizumab (OR 0.81, 95\% CI 0.69-0.95, $P=0.008)$. We also revealed significant associations within some subgroups. The sequential trial analysis showed a truepositive result.
No significant associations were observed between tocilizumab and elevated secondary infection risk, discharge, adverse events, and mechanical ventilation in the overall analysis.

Our data suggests that clinicians should pay attention to tocilizumab therapy as an effective and safe treatment for COVID-19 patients.

\section{DIGITAL FEATURES}

This article is published with digital features, including a summary slide, to facilitate understanding of the article. To view digital features for this article go to https://doi.org/10.6084/ m9.figshare. 14785560 .

\section{INTRODUCTION}

Coronavirus disease 2019 (COVID-19) may be associated with a dysregulated immune response and hyperinflammation, which can lead to or exacerbate acute respiratory distress syndrome (ARDS) and multiple organ failure [1-3]. As the pandemic progressed, there was an unfounded enthusiasm surrounding the use of tocilizumab. However, the clinical efficacy and safety of tocilizumab treatment of COVID-19 patients have been controversial.

Epidemiologic studies and earlier recent retrospective studies have demonstrated that patients infected with SARS-CoV-2 exhibited high plasma levels of circulating interleukin 6 (IL-6), IL-1Ra, IL-1 $\beta$, IL-10, IL-17, IL-18, interferon $\gamma$ (IFN- $\gamma$ ), tumor necrosis factor $\alpha$ (TNF- $\alpha$ ), C-reactive protein (CRP), granulocyte colonystimulating factor (G-CSF), and macrophage colony-stimulating factor (M-CSF), suggesting a rapid activation of the innate immune response [4-11]. Several studies have shown that plasma IL-6 levels are elevated in COVID-19 patients in the intensive care unit (ICU), and they appear to be positively correlated with mortality [12-17]. In an observational study, tocilizumab, an anti-IL-6 receptor monoclonal antibody, has 
been approved for the treatment of various inflammatory diseases and appeared to improve outcomes in COVID-19 patients in several countries [2]. Some recent studies have suggested that tocilizumab may be intensively related to a lower risk of intubation or death in severe and critically ill patients with COVID-19 pneumonia. Since the clinical severity of COVID-19 patients appears to be associated with a cytokines storm and an overproduction of soluble inflammatory mediators, tocilizumab is currently under investigation. Still, the results were inconsistent [18-25].

Amid the shortage of robust evidence regarding the use of tocilizumab in COVID-19 patients, we aimed to evaluate the efficacy and safety of tocilizumab treatment for COVID-19 patients in a case-control study and meta-analysis, which will help inform clinical management of COVID-19 patients.

\section{METHODS}

\section{Patients}

The retrospective case-control study was performed between February to April 2020 and was conducted in Wuhan, China. All participants were admitted to the Huoshenshan Hospital and were confirmed COVID-19 cases according to quantitative real-time polymerase chain reaction (qRT-PCR). We received approval from the ethics committee of Huoshenshan Hospital and conducted the study following the tenets of the Declaration of Helsinki and its amendments.

All participants voluntarily provided written informed consent for sample collection and their subsequent analysis. Intravenous tocilizumab was administered at a dose of $8 \mathrm{mg} / \mathrm{kg}$ body weight (up to $800 \mathrm{mg}$ ), up to twice, $12 \mathrm{~h}$ apart. Matched control patients were retrospectively identified within the electronic information database of Huoshenshan Hospital. The primary endpoint was 60 days' mortality, improvement, and discharge, and critical secondary endpoints were hospital stay, secondary infection, and mechanical ventilation. All participants received standard treatment, including hydroxychloroquine and antiviral therapy, including lopinavir/ritonavir, antimicrobial agents, and corticosteroids.

\section{Study Selection}

We searched PubMed, Web of Science, and MedRxiv using the search terms severe acute respiratory syndrome coronavirus 2, COVID-19, SARS-CoV-2, 2019-nCoV interleukin-6 inhibitors, tocilizumab, and coronavirus for studies published from January 1, 2020, to June 1, 2021 (Fig. 1).

\section{Data Extraction and Verification}

The inclusion criteria of the meta-analysis were: (1) research focus on tocilizumab and COVID19; (2) studies on humans; (3) the number of cases and controls; (4) papers with full text available. The exclusion criteria of the metaanalysis were: (1) review, (2) case report; (3) animal study; (4) incomplete data and unclear outcome effects; (5) repeat of the report.

Specific information about the included studies is listed in Table 3, including (1) the author's last name and country, (2) publication and online time, (3) 3thnicity or race, (4) severity of disease, (5) dose of tocilizumab, (6) study design, (7) journal, (8) case size, (9) number of cases and controls, (10) number of discharges in cases and controls, (11) number of deaths in cases and controls, (12) number of secondary infections in cases and controls, and (13) number of mechanical ventilations in cases and controls. First, three authors (Weijun Jiang, Weiwei Li, and Ying Han) independently screened the studies that met inclusion and exclusion criteria and extracted all data. If two or three of the three agreed, the study was included in the meta-analysis. Next, each of the three authors pulled the data, while the other two cross-checked the data. Disagreements were resolved through review and discussion.

\section{Statistical Analysis}

Categorical variables were summarized as counts and percentages (\%). For the continuous 


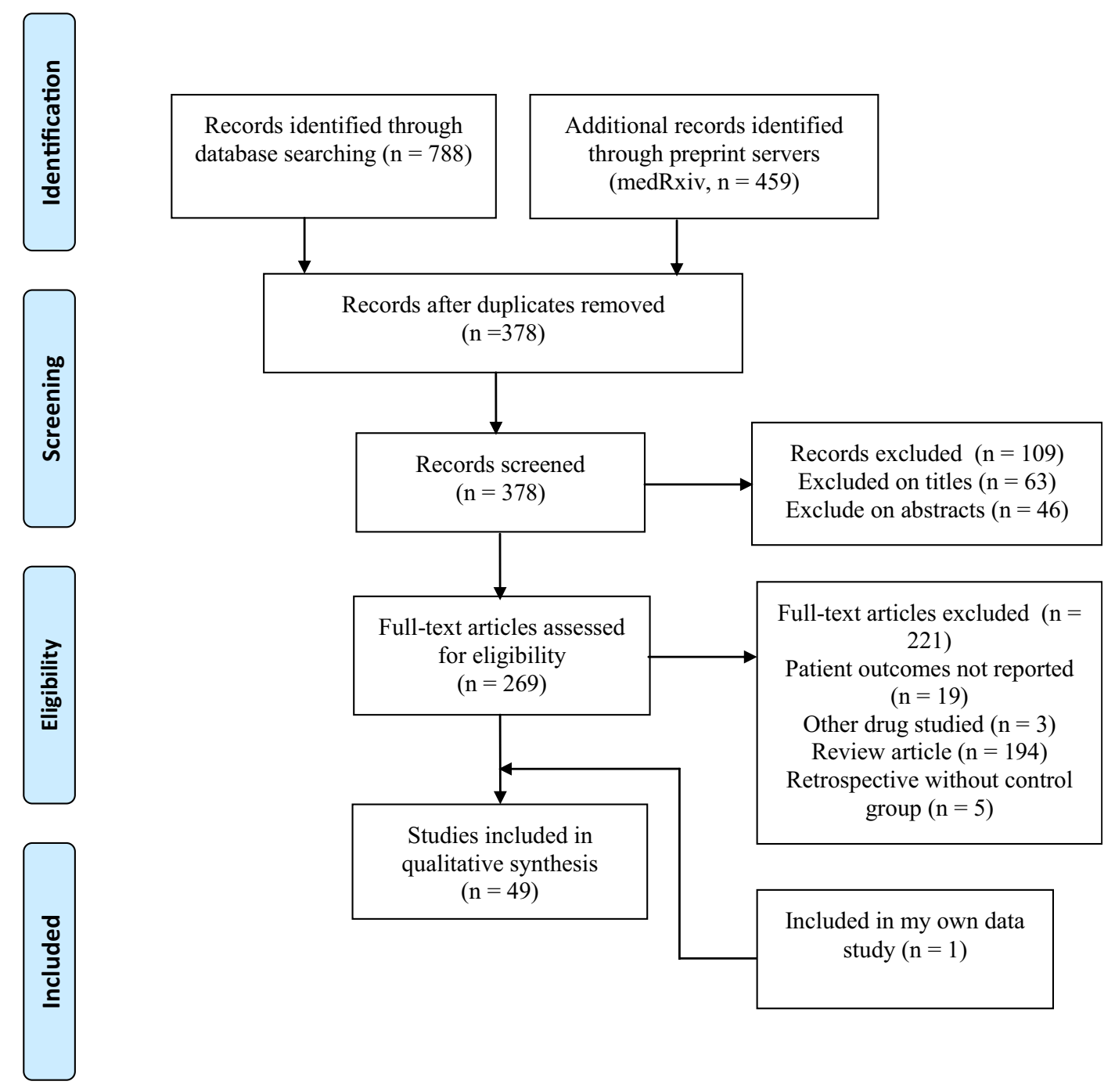

Fig. 1 Flow diagram of the study selection process

measurement, we used the Kolmogorov-Smirnov test to evaluate the distribution type. We also used mean \pm standard deviation (SD) to express the customarily distributed data and otherwise used median and interquartile ranges (IQR) to describe the continuous variables. Continuous variables of different groups of data were compared using independent sample double-tailed $t$-test and Mann-Whitney test. Chi-square and Fisher's exact test was used to compare the frequencies of categorical variables. We used a 1:1 propensity score matching (PSM) analysis to reach patients who underwent tocilizumab with those who did not. We used
SPSS (v.20.0; SPSS Inc., Chicago, IL, USA) to perform statistical analysis.

We used the DerSimonian and Laird method data in the random-effects model and used the Mantel-Haenszel method in the fixed-effects model. $Z$-test was used to determine the pooled odds ratio (OR) statistical significance, with $P$ values $<0.05$ being considered statistically significant. When the $P$ value for heterogeneity was $\leq 0.10$ or $I^{2} \geq 50 \%$, there was heterogeneity between comparative studies. Conversely, if $I^{2}<50 \%$ and the $P$ value for heterogeneity was $>0.10$, this indicated that there was no heterogeneity between comparative studies. We used funnel plots and Egger's linear regression 
test to evaluate publication bias. We used STATA version 11.0 (Stata Corp., College Station, TX, USA) to carry statistical analyses. Similarly, we used novel statistical analysis software, known as trial sequence analysis (TSA), to examine the reliability and conclusiveness of the existing evidence.

\section{RESULTS}

\section{Basic Characteristics and Demographics}

We analyzed a total of 190 participants who were determined to have COVID-19 in the clinical study. Ninety-five patients received standard treatment alone, and 95 received treatment with tocilizumab in addition to standard treatment. Of all patients, 63 (66.32\%) cases and 56 (58.95\%) controls had coexisting conditions, including hypertension, hyperlipidemia, hyperuricemia, diabetes, chronic cardiac disease, cancer, etc.

Interestingly, we found that both hospital stay and secondary infection risk in the case group were significantly higher than in the control group $(P<0.001)$. However, the rate of mortality in the tocilizumab treatment group was lower than that in the standard treatment group, but no statistically significant difference was found. We also found that the mortality rate in tocilizumab treatment groups was significantly lower than in the standard treatment group in the stratified ICU analysis (OR 0.52, 95\% CI $0.44-0.61, P=0.048$ and OR $0.31,95 \%$ CI $0.10-0.99, P=0.044)$. In the subgroup without secondary infection, the tocilizumab treatment group's mortality rate was significantly lower than that in the standard treatment group (OR 0.35, 95\% CI 0.12-1.00, $P=0.040$ ).

However, no significant differences in age, disease severity, rate of cure, and basic disease between the tocilizumab group and standard treatment group were found. Our results showed that tocilizumab treatment did not significantly reduce the rate of COVID-19 mortality and the increased secondary infection risk. Clinicians are advised to use this therapy with caution. The clinical characteristics and demographic data of all participants are shown in Tables 1 and 2.

\section{Study Selection and Characteristics}

The combination of search terms produced all the related articles. A preliminary review of titles and abstracts found 157 articles that required a full manuscript review. According to the inclusion criteria, we selected 49 papers with potential relevance through literature retrieval and screening (Fig. 1). In our metaanalysis, a total of 49 studies, including 6568 cases and 11,660 controls, met the inclusion criteria. Table 3 summarizes the characteristics of the selected studies.

\section{Mortality}

In the overall analysis, we performed a metaanalysis of 49 studies (6568 tolcizumab plus standard treatment COVID-19 patients and 11,660 standard treatment COVID-19 patients) that reported that significantly decreased mortality after patients received tocilizumab (OR $0.81,95 \%$ CI $0.69-0.95, P=0.008$ ). We also revealed significant associations within the mix of race subgroup (OR 0.86, 95\% CI 0.79-0.94, $P=0.001$ ), Caucasian subgroup (OR 0.64, 95\% CI $0.45-0.89, P=0.008$ ), severe subgroup (OR $0.65,95 \%$ CI $0.52-0.82, P=0.001)$, critical subgroup (OR 0.77, 95\% CI 0.66-0.89, $P<0.001$ ), dose of $800 \mathrm{mg}$ subgroup (OR 0.81, 95\% CI $0.70-0.94, P=0.006)$, no specific dose subgroup (OR 0.74, 95\% CI 0.62-0.89, $P=0.001$ ), case-control study subgroup (OR $0.72,95 \%$ CI $0.56-0.91, P=0.006$ ), journal of published subgroup (OR 0.78, 95\% CI $0.64-0.94, P=0.011)$, and case size of $<100$ subgroup (OR 0.77, 95\% CI 0.61-0.98, $P=0.030)$, but not the remaining subgroups. In addition, there was an edge effect in dose of the $\leq 400 \mathrm{mg}$ subgroup (OR 0.66, 95\% CI $0.42-1.03, P=0.067)$ and the multicenter casecontrol subgroup (OR 0.69, 95\% CI 0.45-1.05, $P=0.081$ ). For mortality, the number of patients did not reach the optimal information size. However, the blue cumulative $Z$ curve crossed both the traditional boundary and the 
Table 1 Clinical characteristics of all COVID-19 patients

\begin{tabular}{|c|c|c|c|c|}
\hline Characteristics & All $(N=190)$ & Case $(N=95)$ & Control $(N=95)$ & $P$ value \\
\hline Age $($ mean $\pm S D)$ years & $67.27 \pm 34.51$ & $68.55 \pm 11.50$ & $66 \pm 13.64$ & 0.183 \\
\hline Hospital stay (mean $\pm \mathrm{SD}$ ) days & $24.13 \pm 12.67$ & $12.75 \pm 27.68$ & $11.55 \pm 20.58$ & 0.000 \\
\hline ICU no. (\%) & & & & 0.163 \\
\hline No & $139(71.16 \%)$ & $66(69.47 \%)$ & $73(76.84 \%)$ & \\
\hline Yes & $51(36.84 \%)$ & $29(30.53 \%)$ & $22(23.16 \%)$ & \\
\hline Gender group no. (\%) & & & & 0.765 \\
\hline Male & $120(61.16 \%)$ & $59(62.11 \%)$ & $61(64.21 \%)$ & \\
\hline Female & $70(36.84 \%)$ & $36(37.89 \%)$ & $34(35.79 \%)$ & \\
\hline Basic disease no. (\%) & & & & 0.296 \\
\hline No & $71(37.37 \%)$ & $32(33.68 \%)$ & $39(41.05 \%)$ & \\
\hline Yes & $119(62.63 \%)$ & $63(66.32 \%)$ & $56(58.95 \%)$ & \\
\hline Outcome no. (\%) & & & & 0.134 \\
\hline Cure & $165(86.84 \%)$ & $86(90.53 \%)$ & $79(83.16 \%)$ & \\
\hline Death & $25(13.16 \%)$ & $9(9.47 \%)$ & $16(16.84 \%)$ & \\
\hline Disease severity no. (\%) & & & & 0.789 \\
\hline Moderate & $25(13.16 \%)$ & $13(13.68 \%)$ & $12(12.63 \%)$ & \\
\hline Severe & $108(56.84 \%)$ & $52(54.74 \%)$ & $56(58.95 \%)$ & \\
\hline Critical & $57(30.00 \%)$ & $30(31.58 \%)$ & $27(28.42 \%)$ & \\
\hline \multicolumn{5}{|l|}{ Secondary infection no. (\%) } \\
\hline No & $172(90.53 \%)$ & $79(83.16 \%)$ & $93(97.89 \%)$ & 0.000 \\
\hline Yes & $18(9.47 \%)$ & $16(16.84 \%)$ & $2(2.11 \%)$ & \\
\hline
\end{tabular}

Bold values indicate statistically significant results $\mathrm{SD}$, standard deviation; ICU, intensive care unit

TSA boundary showing that no more tests are needed to reach a positive conclusion in advance. Specific data are summarized in Figs. 2, 3 and Table 4.

\section{Secondary Infection Risk}

No significant associations were observed between tocilizumab and an elevated secondary infection risk in the overall analysis. However, we found significantly increased secondary infection risk after COVID-19 patients received tocilizumab in the dose of $400-800 \mathrm{mg}$ subgroup (OR 1.43, 95\% CI 1.06-1.92, $P=0.018$ ), dose of $\leq 400 \mathrm{mg}$ subgroup (OR 1.92, 95\% CI 1.40-2.63, $P<0.001)$, retrospective observational study subgroup (OR 2.00, 95\% CI $1.15-3.47, P=0.013)$, and case-control study subgroup (OR 1.98, 95\% CI 1.14-3.45, $P=0.016)$. In addition, there was an edge effect in the RCT subgroup (OR 0.79, 95\% CI $0.61-1.03, P=0.079)$. Figure 4 and Table 4 
Table 2 Outcome of tocilizumab treatment COVID-19 patients in stratified analysis

\begin{tabular}{|c|c|c|c|c|c|c|}
\hline & \multirow[t]{2}{*}{ Outcome } & \multicolumn{2}{|l|}{ Tocilizumab } & \multirow[t]{2}{*}{$\operatorname{adjOR}$} & \multirow[t]{2}{*}{ 95\% CIs } & \multirow[t]{2}{*}{$P$} \\
\hline & & Case $(N=95)$ & Control $(N=95)$ & & & \\
\hline \multicolumn{7}{|l|}{ Disease } \\
\hline \multirow[t]{2}{*}{ No } & Cure & 29 & 35 & 0.91 & $0.19-4.38$ & 0.901 \\
\hline & Death & 3 & 4 & & & \\
\hline \multirow[t]{2}{*}{ Yes } & Cure & 57 & 44 & 0.39 & $0.13-1.11$ & 0.069 \\
\hline & Death & 6 & 12 & & & \\
\hline \multicolumn{7}{|l|}{ Severity } \\
\hline \multirow[t]{2}{*}{ Moderate } & Cure & 13 & 12 & & & - \\
\hline & Death & 0 & 0 & & & \\
\hline \multirow[t]{2}{*}{ Severe } & Cure & 52 & 54 & & & 0.103 \\
\hline & Death & 0 & 2 & & & \\
\hline \multirow[t]{2}{*}{ Critical } & Cure & 21 & 13 & 0.40 & $0.13-1.18$ & 0.092 \\
\hline & Death & 9 & 14 & & & \\
\hline \multicolumn{7}{|l|}{$\mathrm{ICU}$} \\
\hline \multirow[t]{2}{*}{ No } & Cure & 66 & 70 & 0.52 & $0.44-0.61$ & 0.048 \\
\hline & Death & 0 & 3 & & & \\
\hline \multirow[t]{2}{*}{ Yes } & Cure & 20 & 9 & 0.31 & $0.10-0.99$ & 0.044 \\
\hline & Death & 9 & 13 & & & \\
\hline \multicolumn{7}{|l|}{ Gender } \\
\hline \multirow[t]{2}{*}{ Female } & Cure & 32 & 30 & 0.94 & $0.22-4.09$ & 0.932 \\
\hline & Death & 4 & 4 & & & \\
\hline \multirow[t]{2}{*}{ Male } & Cure & 54 & 49 & 0.38 & $0.12-1.12$ & 0.075 \\
\hline & Death & 5 & 12 & & & \\
\hline \multicolumn{7}{|c|}{ Secondary infection } \\
\hline \multirow[t]{2}{*}{ No } & Cure & 74 & 78 & 0.35 & $0.12-1.00$ & 0.040 \\
\hline & Death & 5 & 15 & & & \\
\hline \multirow[t]{2}{*}{ Yes } & Cure & 12 & 1 & 0.33 & $0.02-6.65$ & 0.478 \\
\hline & Death & 4 & 1 & & & \\
\hline
\end{tabular}

Bold values indicate statistically significant results

ICU, intensive care unit; $P_{\mathrm{h}}, P$ value of heterogeneity, $P$ value of $Q$-test for the heterogeneity test; OR, odds ratio; CI, confidence interval 


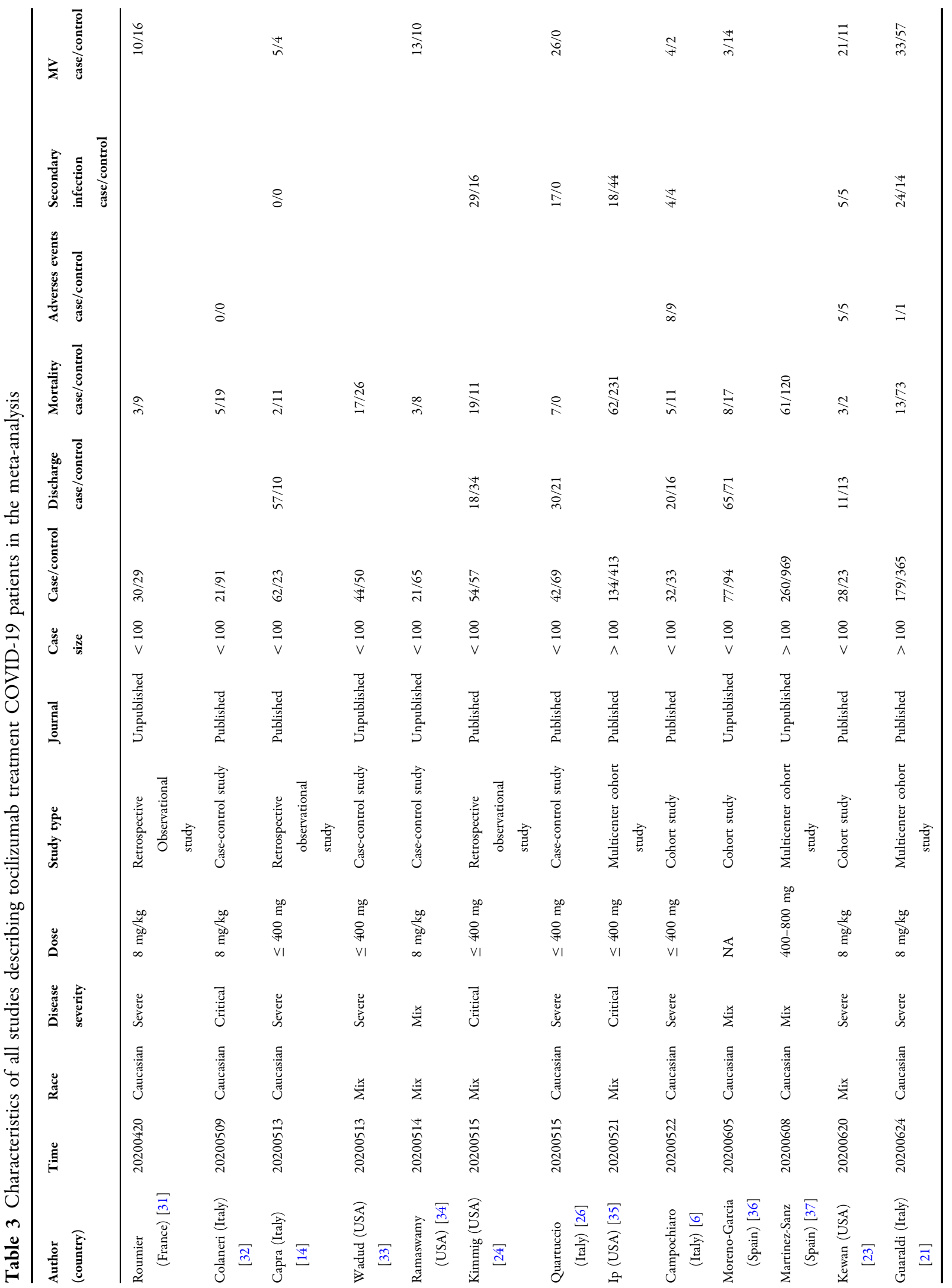




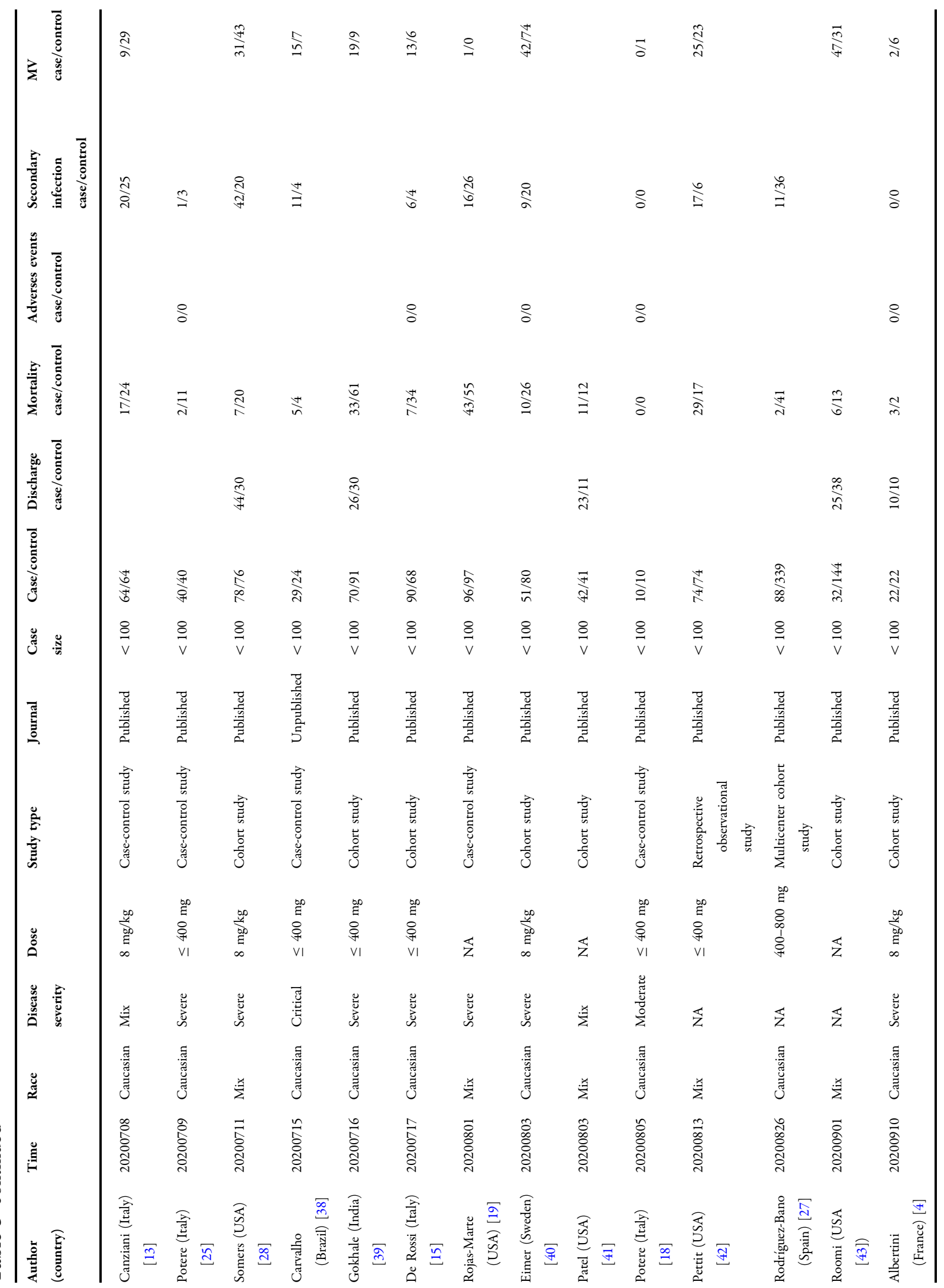




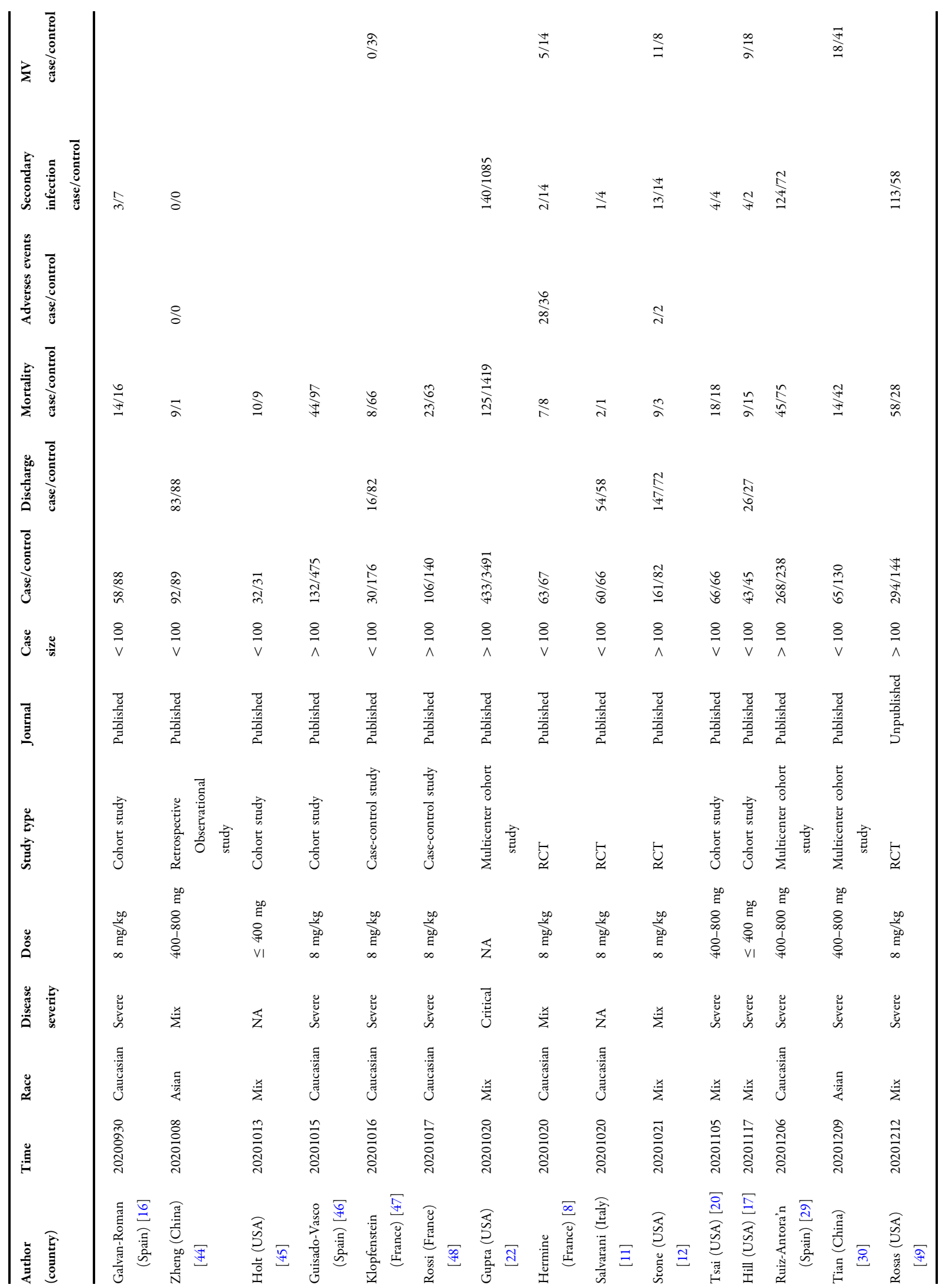




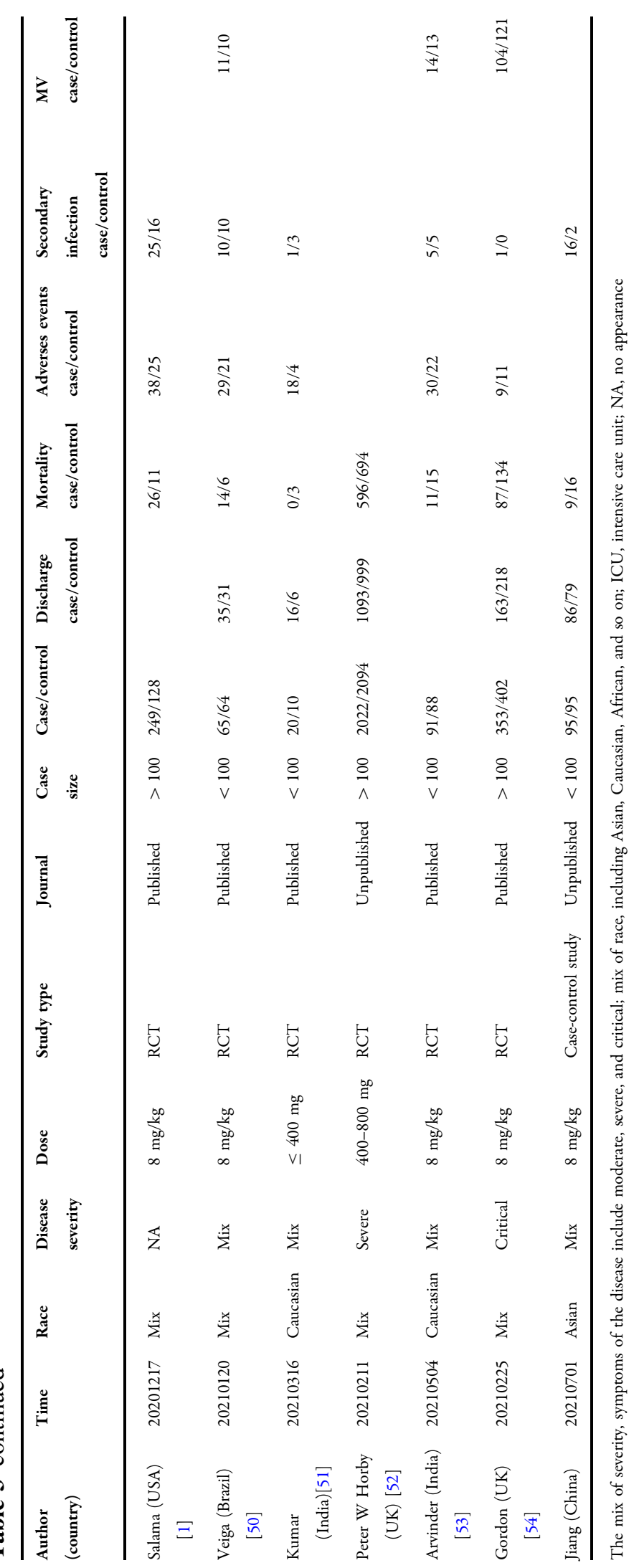




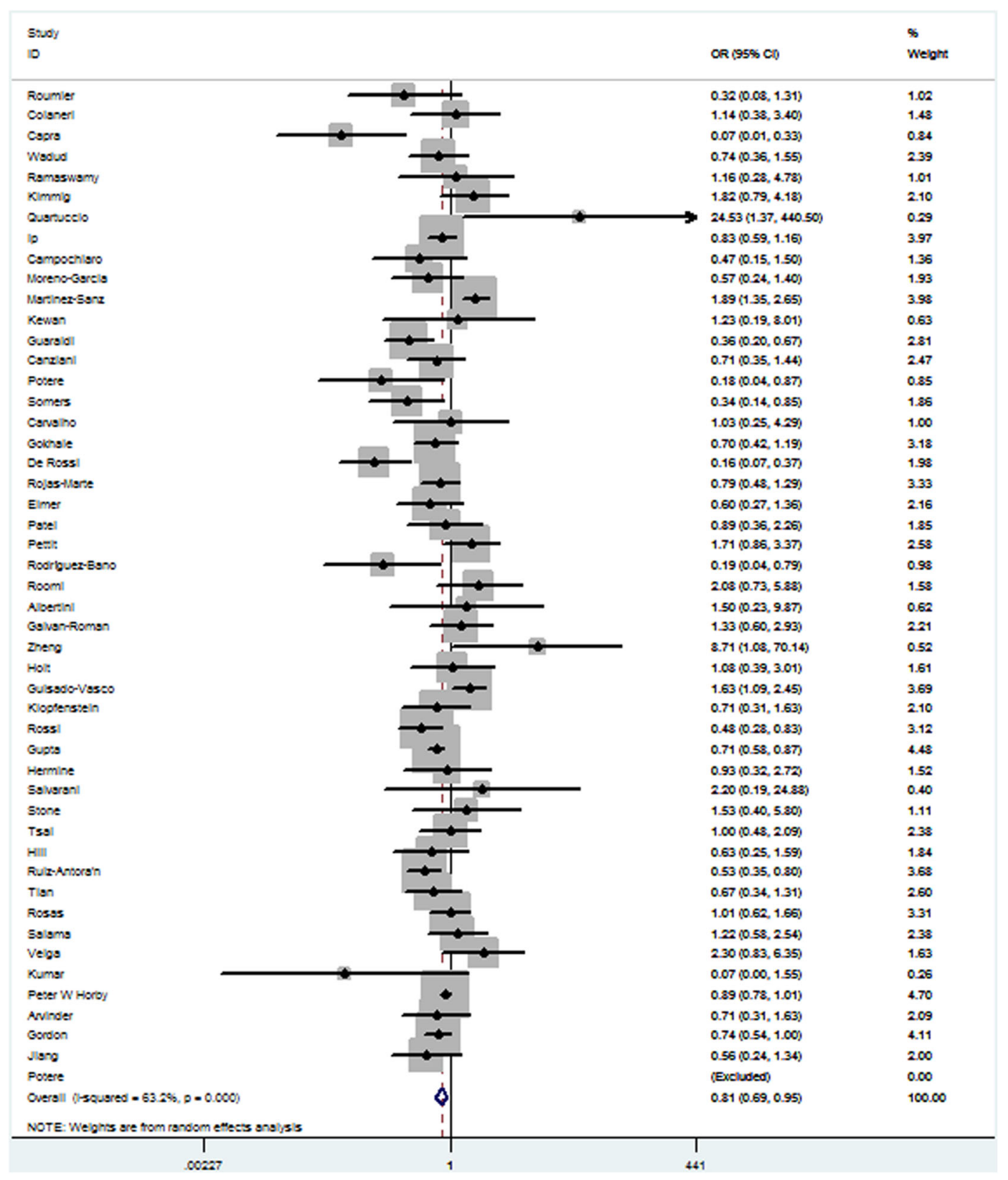

Fig. 2 Forest plot of mortality and tocilizumab treatment COVID-19 patients

summarize the data associated with tocilizumab and secondary infection risk.

\section{Discharge}

We also failed to find significantly increased discharge after COVID-19 patients received tocilizumab in the overall analysis (OR 1.13, 95\% CI $0.98-1.32, \quad P=0.100)$. We revealed significantly increased discharge in tocilizumab treatment COVID-19 patients in the cohort study subgroup (OR 1.32, 95\% CI 1.06-1.65, $P=0.014)$. We revealed significantly decreased discharge in tocilizumab treatment COVID-19 patients in the dose of $400-800 \mathrm{mg}$ subgroup (OR 0.88, 95\% CI 0.80-0.98, $P=0.018$ ). However, we also found an edge effect in Caucasian subgroup analysis (OR 1.24, 95\% CI 1.00-1.55, 


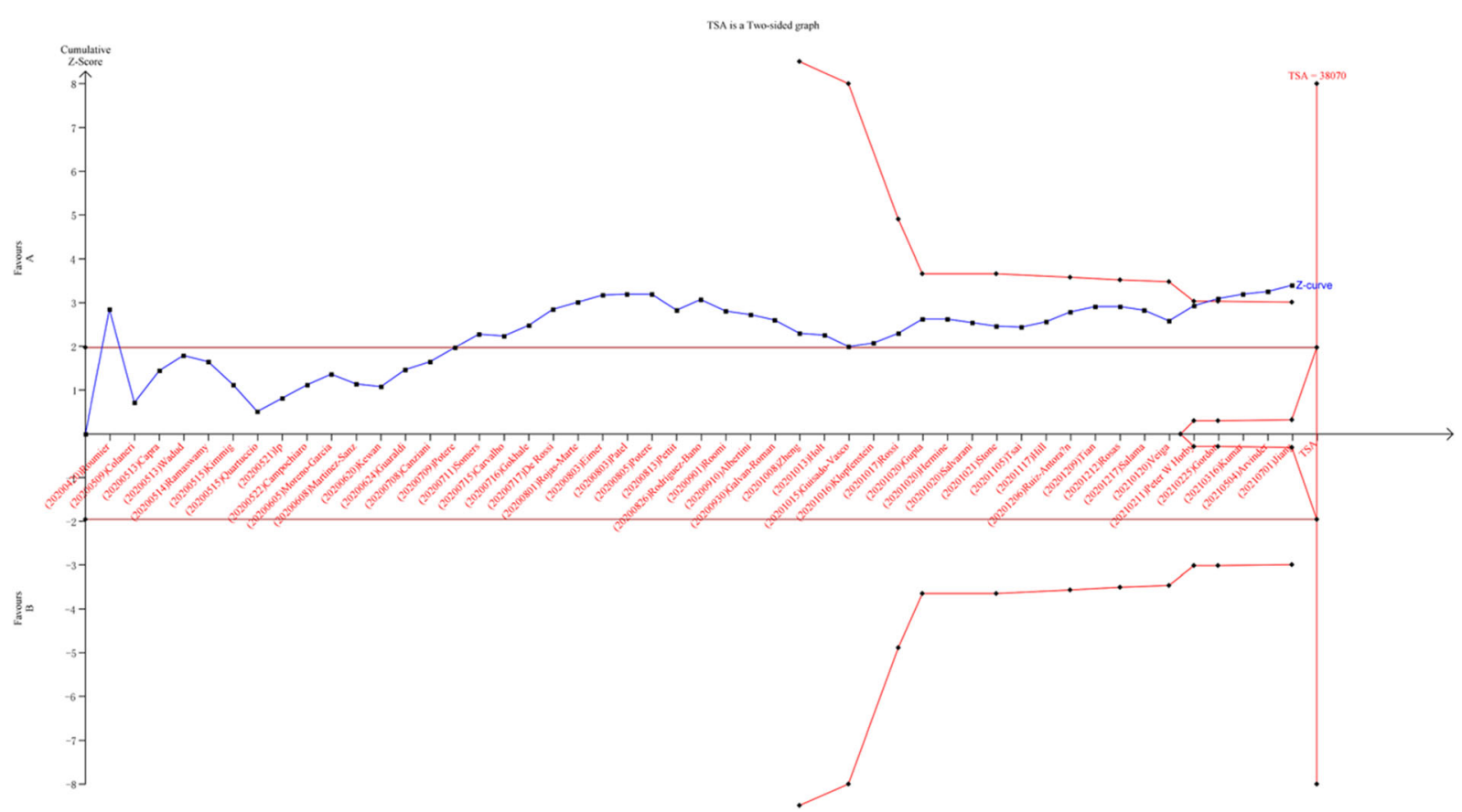

Fig. 3 Trial sequential analysis of mortality and tocilizumab treatment COVID-19 patients

$P=0.051)$, the no specific dose subgroup (OR $1.82,95 \%$ CI $0.95-3.47, P=0.069)$, case-control study subgroup (OR 1.37, 95\% CI 1.00-1.89, $P=0.051$ ), journal of unpublished subgroup (OR 0.91, 95\% CI 0.0.82-1.00, $P=0.055$ ), journal of published subgroup (OR 1.18, 95\% CI $0.99-1.41, P=0.060)$, and case size of $<100$ subgroup (OR 1.19, 95\% CI 0.99-1.44, $P=0.060)$. Specific data are summarized in Fig. 4 and Table 5.

\section{Adverse Events and Mechanical Ventilation}

No significant association was found between tocilizumab and adverse events and mechanical ventilation in COVID-19 patients in the overall analysis. We found significantly increased adverse events risk in tocilizumab treatment COVID-19 patients in the RCT subgroup (OR 1.32 , 95\% CI 1.00-1.74, $P=0.049$ ). Subgroup analyses were performed to clarify the effects of potential ethnicity, disease severity, drug dose, study type, and case size and whether the article is officially published. There was no significant association between tocilizumab treatment and estimated risk of adverse events and mechanical ventilation, as shown in Fig. 4 and Table 5.

\section{Publication Bias and Sensitivity Analysis}

We performed Egger's test and Begg's funnel plot to assess the publication bias of the metaanalysis. We also conducted sensitivity analysis by omitting one study at a time when calculating summary results. Although the size of the sample of cases in all included studies varied, corresponding pooled ORs and 95\%CIs did not qualitatively change with or without studies on small pieces.

\section{DISCUSSION}

The World Health Organization (WHO) announced COVID-19 may progress to a pandemic associated with substantial morbidity and mortality and is a public health emergency of globalized concern as of 1 February 2020 $[1,3,12]$. At the time of this writing, over 174 million laboratory-diagnosed COVID-19 patients had been reported spanning 212 


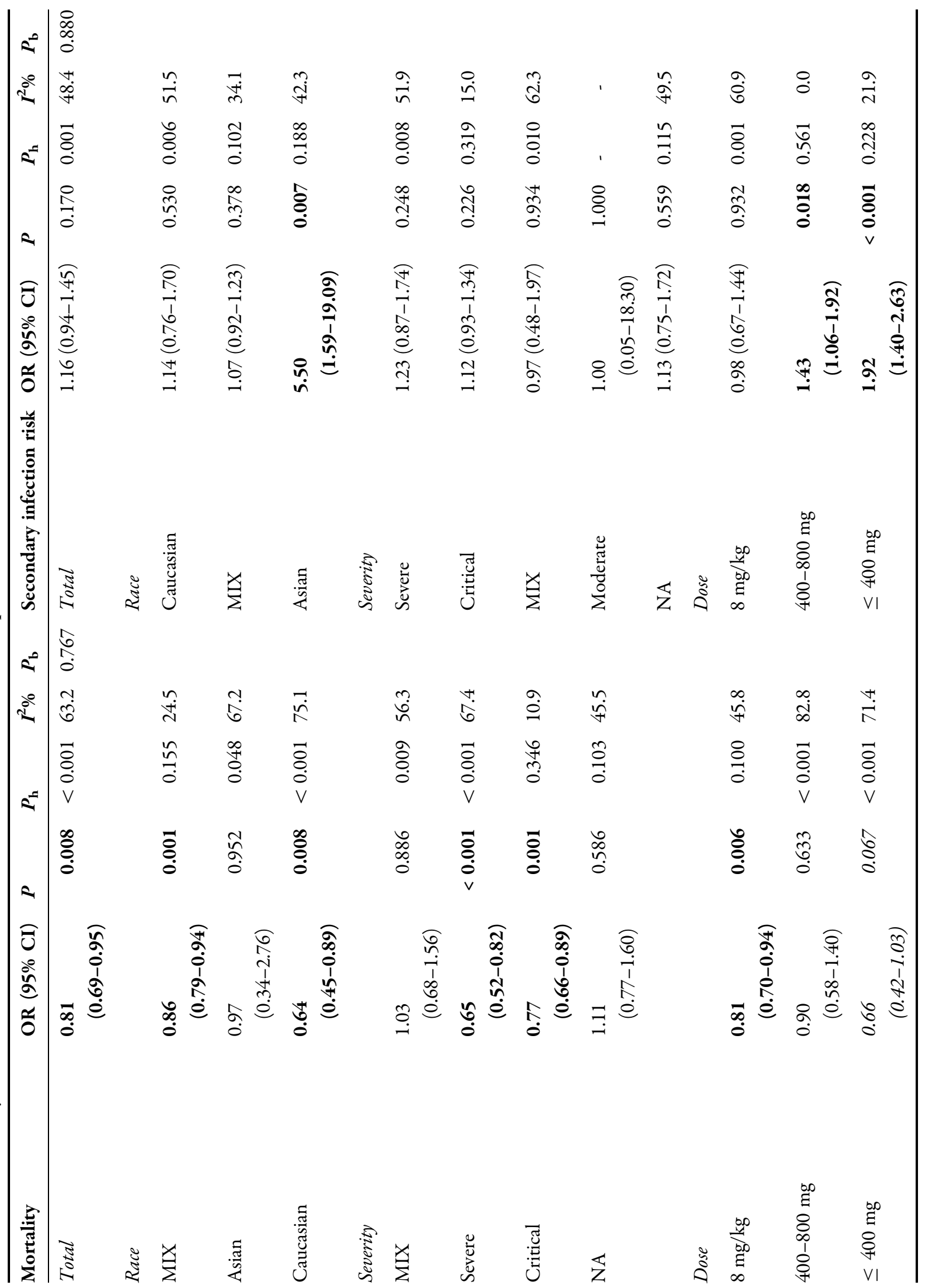




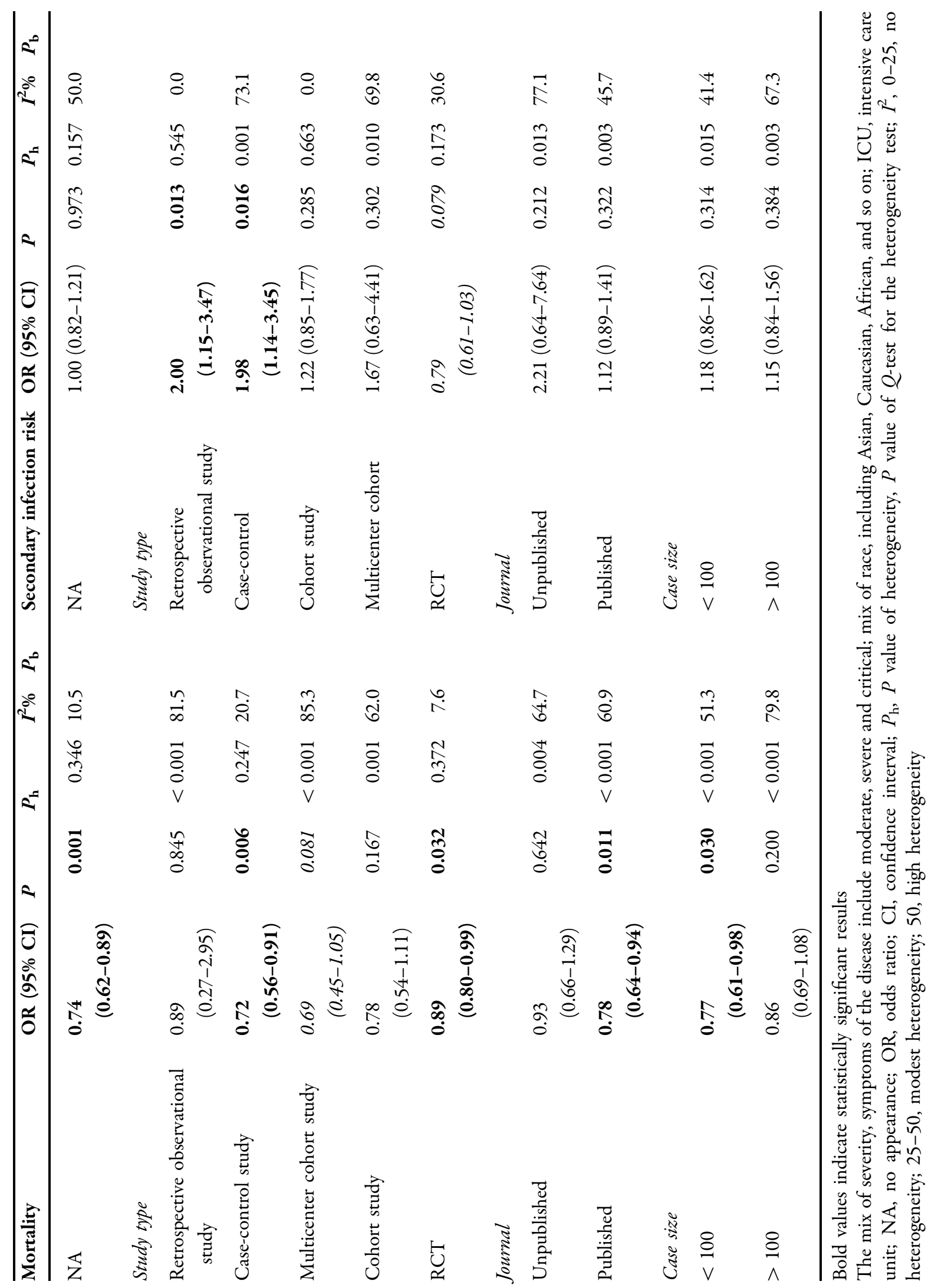


a

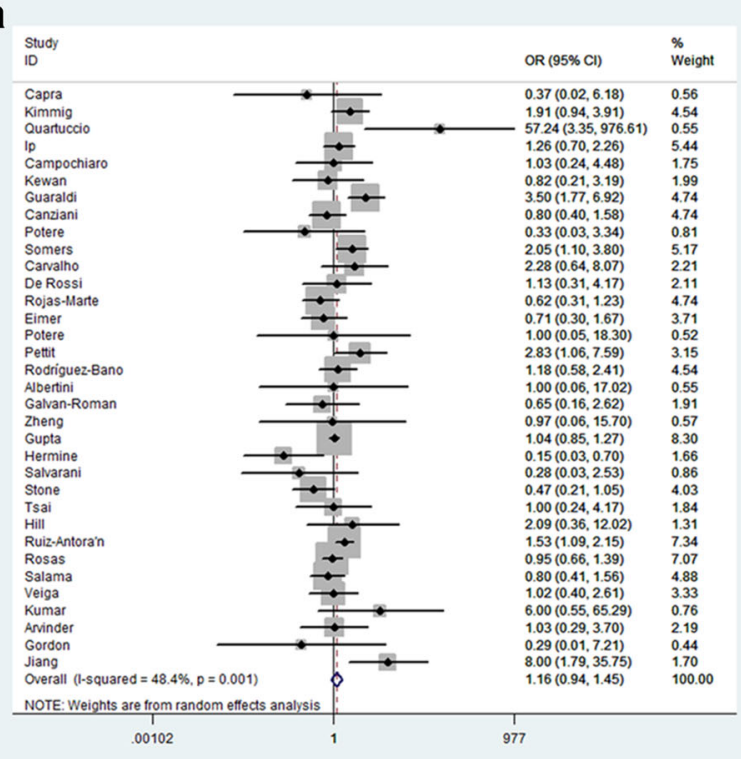

C

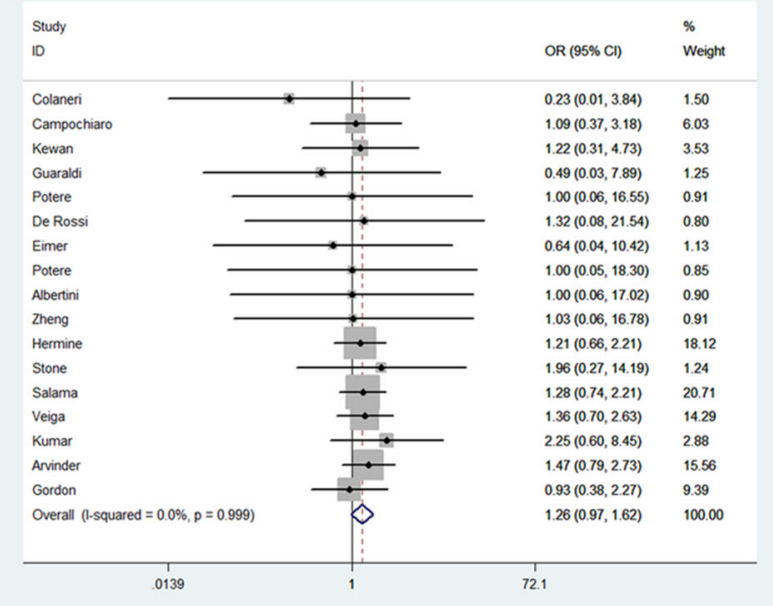

Fig. 4 Forest plot of safety of tocilizumab treatment COVID-19 patients. a Forest plot of tocilizumab and secondary infection risk in COVID-19 patients. b Forest plot of tocilizumab and discharge in COVID-19 patients.

countries and regions and contributing to $>$ $3,730,000$ death.

SARS-CoV-2 brings about a broad spectrum of clinical manifestations, ranging from asymptomatic or paucisymptomatic forms (with malaise, myalgia, dry cough, and fever) to full-blown viral pneumonia ARDS, multiorgan failure, and death $[2,25]$. The serum cytokine profiles of some moderate to severe COVID-19 patients overlap with secondary b

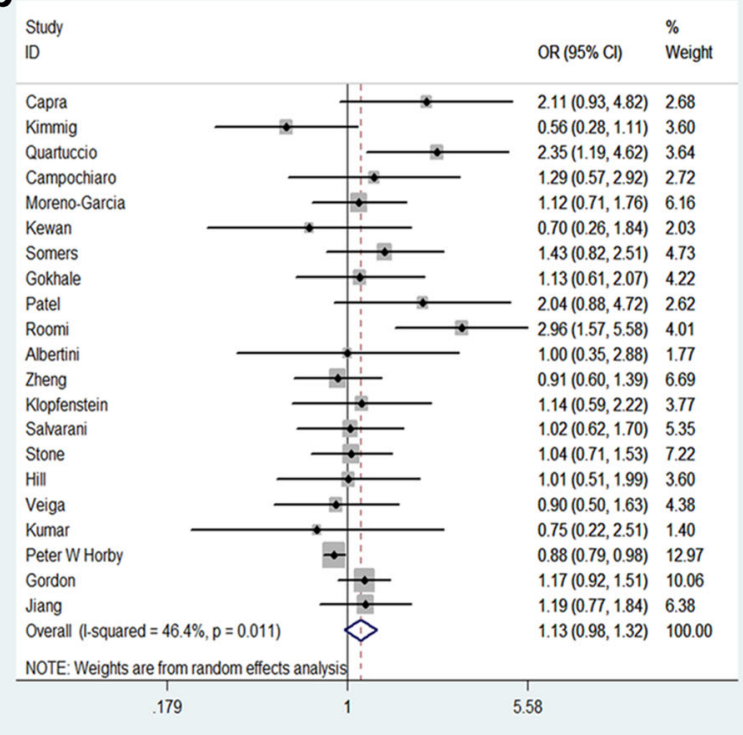

d

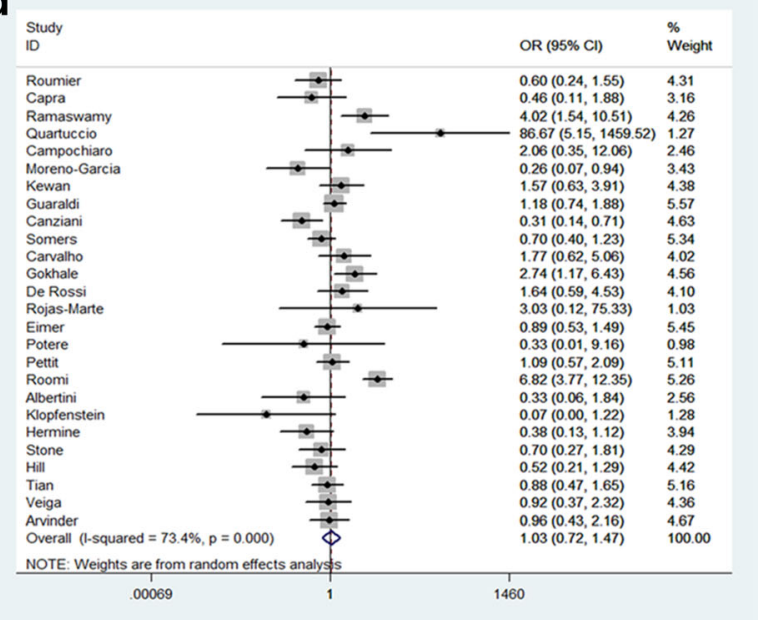

c Forest plot of tocilizumab and adverse events in COVID-19 patients. d Forest plot of tocilizumab and mechanical ventilation in COVID-19 patients

hemophagocytic lymphocytosis (sHLH) and macrophage activation syndrome (MAS). Viruses are known as solid triggers of MAS/sHLH, and serum ferritin levels are associated with mortality in MAS and COVID-19 patients. Endogenous IL-1 is a proinflammatory cytokine that induces the production of IL- 6 by macrophages and monocytes and is elevated in MAS, COVID-19 disease, and other diseases, such as severe chimerical antigen receptor T-cell (CAR- 


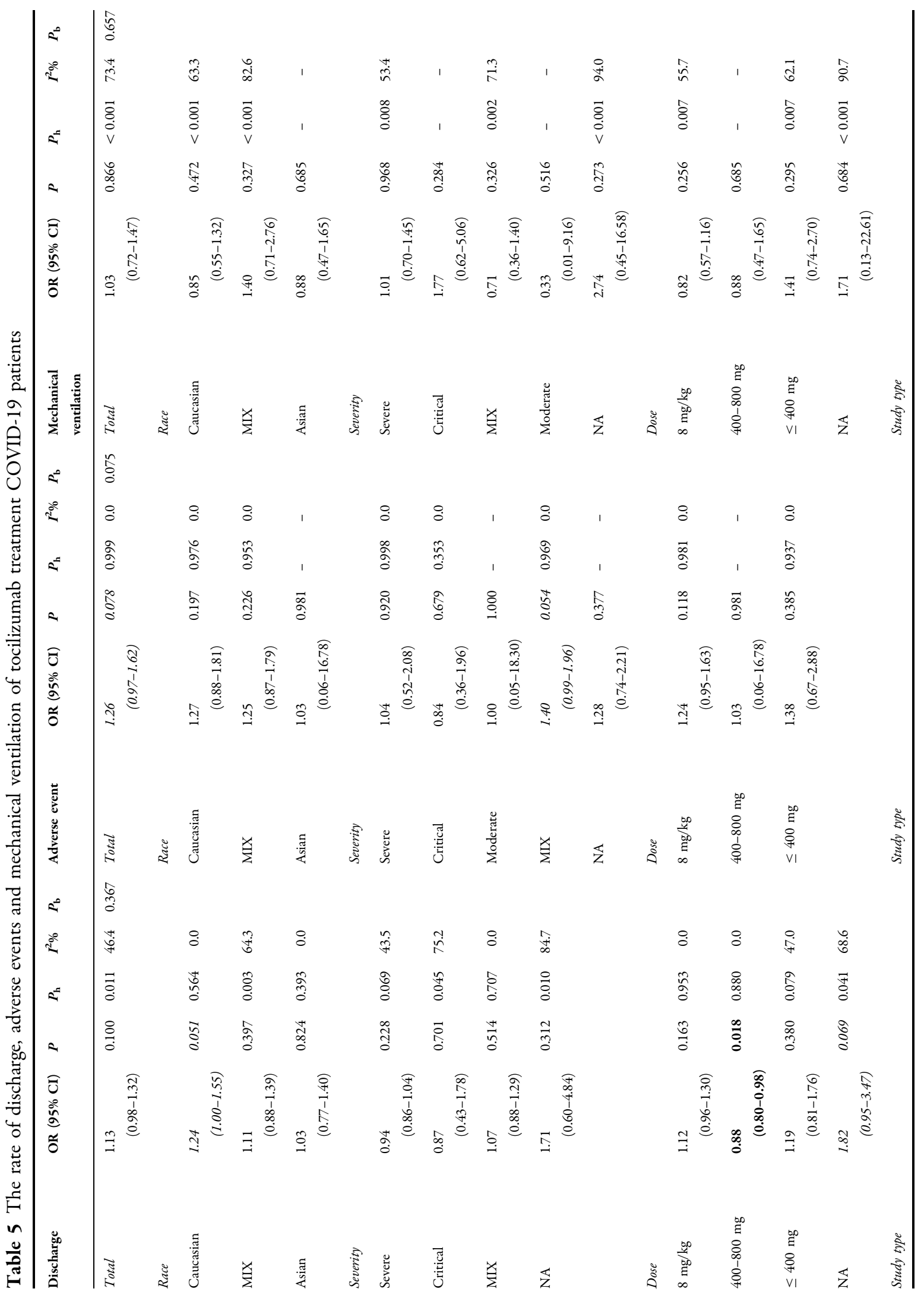




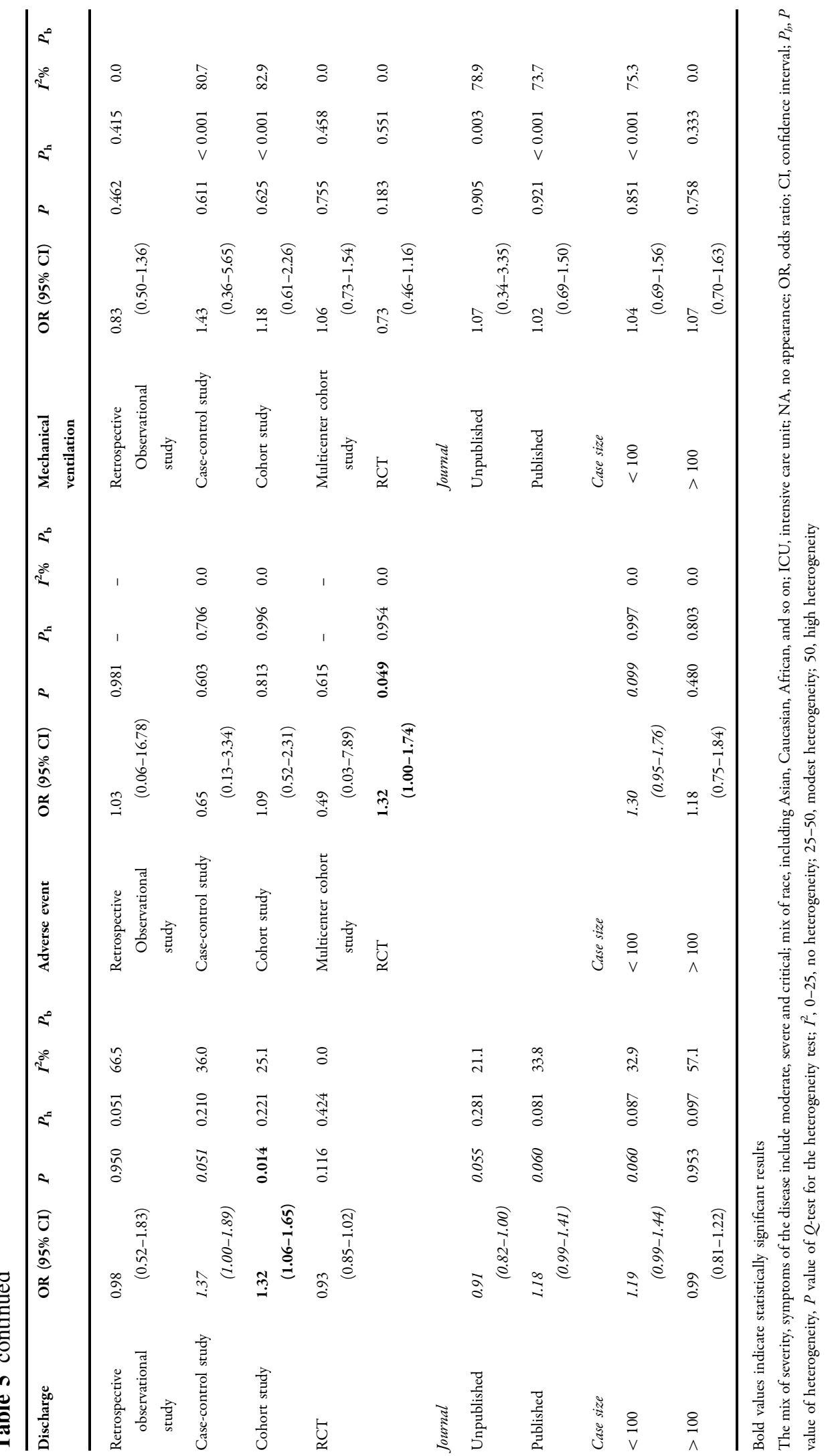


T)-mediated cytokine release syndrome (CRS) [2, 26-28].

IL-6 inhibitors or their receptor inhibitors have been successfully treated with other cytokine storm syndromes or CRS secondary to CAR T-cell therapies. We already have several drug options available, including IL-6 inhibitors (siltuximab, sirukumab, clazakizumab) and IL-6 receptor inhibitors (tocilizumab, sarilumab). It is noteworthy that tocilizumab has been officially included in the National Health Commission of the People's Republic of China COVID-19 Guidelines for diagnosis and treatment program (7th edition) since March 2020: "Tocilizumab can be used in COVID-19 patients with extensive ground-glass lesions opacity in bilateral lungs or critically ill COVID-19 patients, who have elevated laboratory detected serum IL-6 levels" [2]. The Infectious Diseases Society of America (IDSA) has also recently published guidelines and suggested that tocilizumab should only be used in clinical trials of hospitalized COVID-19 patients because of the lack of reliable clinical treatment data [2].

The Infectious Diseases Society of America (IDSA) has also recently published guidelines and suggested that tocilizumab should only be used in clinical trials of hospitalized COVID-19 patients because of the lack of reliable clinical treatment data $[1,11,12]$. However, several retrospective observational studies have found that, in addition to standard treatment, tocilizumab is a safe and promising treatment to prevent disease progression in hospitalized COVID-19 patients with moderate and hyperinflammation [13-16, 28-30]. Our case-control study also found a lower mortality rate in the tocilizumab treatment group than in the standard treatment group (9.47\% versus $16.84 \%)$, but the results were not statistically significant. However, we found that tocilizumab significantly decreased mortality and increased discharge in COVID-19 patients but no increased secondary infection risk, adverse event, and mechanical ventilation in a meta-analysis of 49 studies (6568 cases and 11,660 controls). We additionally found similar results in several subgroups. Therefore, our data suggest that clinicians should pay attention to tocilizumab therapy as an effective and safe treatment for COVID-19 patients.

Some limitations of the study need to be noted. First, in the absence of clinical test data of every patient, it is not clear which patients with high clinical indicators will benefit most when treated with tocilizumab. Second, subgroup analyses involved relatively small groups, which may not provide sufficient statistical power to explore accurate correlations. Third, every doctor has a different treatment for clinical diagnostics, which would allow for adjustment by other factors. Finally, the inclusion of zero-event trials can sometimes reduce effect size estimates and narrow confidence intervals.

\section{CONCLUSION}

To our knowledge, it is the systematic review and meta-analysis to investigate the efficacy and safety of tocilizumab treatment in COVID19 patients in the the biggest sample size. Tocilizumab significantly decreased mortality in COVID-19 patients but no increased discharge, secondary infection risk, adverse event, and mechanical ventilation in a meta-analysis. Our data suggest that clinicians should pay attention to tocilizumab therapy as an effective and safe treatment for COVID-19 patients.

\section{ACKNOWLEDGEMENTS}

I am very grateful to my wife for her help and support. We also appreciate the valuable comments from other members of our laboratory.

Funding. Our work was supported by the Key Foundation of Wuhan Huoshenshan Hospital (2020[18]), Scientific Research Project of Jiangsu Commission of Health (H2019065), Medical Innovation Project of Logistics Service (18JS005), and Key Research \& Development Program of Jiangsu Province (BE2018713). The Journal's Rapid Service Fee was funded by the authors.

Authorship. All named authors meet the International Committee of Medical Journal 
Editors (ICMJE) criteria for authorship for this article, take responsibility for the integrity of the work as a whole, and have given their approval for this version to be published.

Authorship contributions. All authors reviewed and approved the final manuscript. Weijun Jiang wrote and revised the paper. Weijun Jiang, Qiuyue Wu, and Xinyi Xia conceived and designed the experiments. Weijun Jiang, Ying Han, and Weiwei Li performed publication searches and selection. Weijun Jiang and Peiran Zhu contributed materials/analysis tools. Weijun Jiang, Yang Yang, and Yanju Guo prepared the figures. Weijun Jiang, Jing Zhang, and Tao Luo analyzed the data.

Disclosures. All authors declare that they have no competing interests in the work.

Compliance with Ethics Guidelines. All participants voluntarily provided written informed consent for sample collection and their subsequent analysis. We have the approval of the ethics committee of Huoshenshan Hospital and have conducted it following the tenets of the Declaration of Helsinki and its amendments.

Data Availability. The datasets generated during and analyzed during the current study are available from the corresponding author on reasonable request.

Open Access. This article is licensed under a Creative Commons Attribution-NonCommercial 4.0 International License, which permits any non-commercial use, sharing, adaptation, distribution and reproduction in any medium or format, as long as you give appropriate credit to the original author(s) and the source, provide a link to the Creative Commons licence, and indicate if changes were made. The images or other third party material in this article are included in the article's Creative Commons licence, unless indicated otherwise in a credit line to the material. If material is not included in the article's Creative Commons licence and your intended use is not permitted by statutory regulation or exceeds the permitted use, you will need to obtain permission directly from the copyright holder. To view a copy of this licence, visit http://creativecommons.org/licenses/by$\mathrm{nc} / 4.0 /$.

\section{REFERENCES}

1. Salama C, Han J, Yau L, et al. Tocilizumab in patients hospitalized with covid-19 pneumonia. N Engl J Med. 2020;384:20-30.

2. Jamilloux Y, Henry $T$, Belot A, et al. Should we stimulate or suppress immune responses in COVID19? Cytokine and anti-cytokine interventions. Autoimmun Rev. 2020;19:102567.

3. Jiang W, Li W, Xiong L, et al. Clinical efficacy of convalescent plasma therapy on treating COVID-19 patients: Evidence from matched study and a metaanalysis. Clin Transl Med. 2020;10:e259.

4. Albertini L, Soletchnik M, Razurel A, et al. Observational study on off-label use of tocilizumab in patients with severe COVID-19. Eur J Hosp Pharm. $2021 ; 28: 22-7$

5. Cauchois R, Koubi M, Delarbre D, et al. Early IL-1 receptor blockade in severe inflammatory respiratory failure complicating COVID-19. Proc Natl Acad Sci USA. 2020;117:18951-3.

6. Campochiaro C, Della-Torre E, Cavalli G, et al. Efficacy and safety of tocilizumab in severe COVID19 patients: a single-centre retrospective cohort study. Eur J Intern Med. 2020;76:43-9.

7. Della-Torre E, Campochiaro C, Cavalli G, et al. Interleukin-6 blockade with sarilumab in severe COVID-19 pneumonia with systemic hyperinflammation: an open-label cohort study. Ann Rheum Dis. 2020;79:1277-85.

8. Hermine $\mathrm{O}$, Mariette $\mathrm{X}$, Tharaux PL, Resche-Rigon M, Porcher R, Ravaud P. Effect of tocilizumab vs usual care in adults hospitalized with COVID-19 and moderate or severe pneumonia: a randomized clinical trial. JAMA Intern Med. 2020;181:32-40.

9. Huet $\mathrm{T}$, Beaussier $\mathrm{H}$, Voisin $\mathrm{O}$, et al. Anakinra for severe forms of COVID-19: a cohort study. Lancet Rheumatol. 2020;2:e393-400.

10. Kooistra EJ, Waalders NJB, Grondman I, et al. Anakinra treatment in critically ill COVID-19 patients: a prospective cohort study. Crit Care. 2020;24:688. 
11. Salvarani C, Dolci G, Massari M, et al. Effect of tocilizumab vs standard care on clinical worsening in patients hospitalized with COVID-19 pneumonia: a randomized clinical trial. JAMA Intern Med. 2020;181:24-31.

12. Stone JH, Frigault MJ, Serling-Boyd NJ, et al. Efficacy of tocilizumab in patients hospitalized with covid-19. N Engl J Med. 2020;383:2333-44.

13. Canziani LM, Trovati S, Brunetta E, et al. Interleukin-6 receptor blocking with intravenous tocilizumab in COVID-19 severe acute respiratory distress syndrome: a retrospective case-control survival analysis of 128 patients. J Autoimmun. 2020;114:102511.

14. Capra R, De Rossi N, Mattioli F, et al. Impact of low dose tocilizumab on mortality rate in patients with COVID-19 related pneumonia. Eur J Intern Med. 2020;76:31-5.

15. De Rossi N, Scarpazza C, Filippini C, et al. Early use of low dose tocilizumab in patients with COVID-19: a retrospective cohort study with a complete follow-up. EClinicalMedicine. 2020;25:100459.

16. Galván-Román JM, Rodríguez-García SC, Roy-Vallejo E, et al. IL-6 serum levels predict severity and response to tocilizumab in COVID-19: an observational study. J Allergy Clin Immunol. 2020;147: 72-80.e8.

17. Hill JA, Menon MP, Dhanireddy S, et al. Tocilizumab in hospitalized patients with COVID-19: clinical outcomes, inflammatory marker kinetics, and safety. J Med Virol. 2020;93:2270-80.

18. Potere N, Di Nisio M, Rizzo G, et al. Low-dose subcutaneous tocilizumab to prevent disease progression in patients with moderate COVID-19 pneumonia and hyperinflammation. Int $\mathrm{J}$ Infect Dis. 2020;100:421-4.

19. Rojas-Marte G, Khalid M, Mukhtar O, et al. Outcomes in patients with severe COVID-19 disease treated with tocilizumab: a case-controlled study. QJM. 2020;113:546-50.

20. Tsai A, Diawara O, Nahass RG, Brunetti L. Impact of tocilizumab administration on mortality in severe COVID-19. Sci Rep. 2020;10:19131.

21. Guaraldi G, Meschiari M, Cozzi-Lepri A, et al. Tocilizumab in patients with severe COVID-19: a retrospective cohort study. Lancet Rheumatol. 2020;2:e474-84.

22. Gupta S, Wang W, Hayek SS, et al. Association between early treatment with tocilizumab and mortality among critically ill patients with COVID19. JAMA Intern Med. 2020;181(1):41-51.
23. Kewan T, Covut F, Al-Jaghbeer MJ, Rose L, Gopalakrishna KV, Akbik B. Tocilizumab for treatment of patients with severe COVID-19: a retrospective cohort study. EClinicalMedicine. 2020;24: 100418.

24. Kimmig LM, Wu D, Gold M, et al. IL-6 inhibition in critically ill covid-19 patients is associated with increased secondary infections. Front Med (Lausanne). 2020;7:583897.

25. Potere N, Di Nisio M, Cibelli D, et al. Interleukin-6 receptor blockade with subcutaneous tocilizumab in severe COVID-19 pneumonia and hyperinflammation: a case-control study. Ann Rheum Dis. 2020;80:1-2.

26. Quartuccio L, Sonaglia A, McGonagle D, et al. Profiling COVID-19 pneumonia progressing into the cytokine storm syndrome: results from a single Italian Centre study on tocilizumab versus standard of care. J Clin Virol. 2020;129:104444.

27. Rodríguez-Baño J, Pachón J, Carratalà J, et al. Treatment with tocilizumab or corticosteroids for COVID-19 patients with hyperinflammatory state: a multicentre cohort study (SAM-COVID-19). Clin Microbiol Infect. 2020;27:244-52.

28. Somers EC, Eschenauer GA, Troost JP, et al. Tocilizumab for treatment of mechanically ventilated patients with COVID-19. Clin Infect Dis. 2020; ciaa954.

29. Ruiz-Antorán B, Sancho-López A, Torres F, et al. Combination of tocilizumab and steroids to improve mortality in patients with severe COVID19 infection: a Spanish, multicenter, cohort study. Infect Dis Ther. 202010(1):347-62.

30. Tian J, Zhang M, Jin M, et al. Repurposed tocilizumab in patients with severe COVID-19. J Immunol. 2020;206:599-606.

31. Roumier M, Paule R, Groh M, Vallée A, Ackermann F. Interleukin-6 blockade for severe COVID-19. medRxiv. 2020:2020.04.20.20061861.

32. Colaneri M, Bogliolo L, Valsecchi P, et al. Tocilizumab for treatment of severe COVID-19 patients: preliminary results from SMAtteo COvid19 REgistry (SMACORE). Microorganisms. 2020;8:695.

33. Wadud N, Ahmed N, Shergil M, et al. Improved survival outcome in SARs-CoV-2 (COVID-19) acute respiratory distress syndrome patients with tocilizumab administration. medRxiv. 2020:2020.05.13. 20100081.

34. Ramaswamy M, Mannam P, Comer R, Sinclair E, McQuaid DB, Schmidt ML. Off-label real world experience using tocilizumab for patients 
hospitalized with COVID-19 disease in a regional community health system: a case-control study. medRxiv. 2020:2020.05.14.20099234.

35. Ip A, Berry DA, Hansen E, et al. Hydroxychloroquine and tocilizumab therapy in COVID-19 patients-An observational study. PLoS One. 2020;15:e0237693.

36. Moreno-García E, Rico V, Albiach L, et al. Tocilizumab is associated with reduced risk of ICU admission and mortality in patients with SARSCoV-2 infection. medRxiv. 2020:2020.06.05. 20113738.

37. Martínez-Sanz J, Muriel A, Ron R, et al. Effects of tocilizumab on mortality in hospitalized patients with COVID-19: a multicenter cohort study. medRxiv. 2020:2020.06.08.20125245.

38. Carvalho V, Turon R, Gonçalves B, Ceotto VF, Kurtz P, Righy C. Effects of tocilizumab in critically ill patients with COVID-19: a quasi-experimental study. medRxiv. 2020:2020.07.13.20149328.

39. Gokhale Y, Mehta R, Karnik N, Kulkarni U, Gokhale S. Tocilizumab improves survival in patients with persistent hypoxia in severe COVID-19 pneumonia. EClinicalMedicine. 2020;24:100467.

40. Eimer J, Vesterbacka J, Svensson AK, et al. Tocilizumab shortens time on mechanical ventilation and length of hospital stay in patients with severe COVID-19: a retrospective cohort study. J Intern Med. 2020;289:434-6.

41. Patel K, Gooley TA, Bailey N, et al. Use of the IL-6R antagonist tocilizumab in hospitalized COVID-19 patients. J Intern Med. 2021;289:430-3.

42. Pettit NN, Nguyen CT, Mutlu GM, et al. Late onset infectious complications and safety of tocilizumab in the management of COVID-19. J Med Virol. 2020;93:1459-64.

43. Roomi S, Ullah W, Ahmed F, et al. efficacy of hydroxychloroquine and tocilizumab in patients with COVID-19: Single-C. J Med Internet Res. 2020;22:e21758.

44. Zheng KL, Xu Y, Guo YF, et al. Efficacy and safety of tocilizumab in COVID-19 patients. Aging (Albany NY). 2020;12:18878-88.

45. Holt GE, Batra M, Murthi M, et al. Lack of tocilizumab effect on mortality in COVID19 patients. Sci Rep. 2020;10:17100.
46. Guisado-Vasco P, Valderas-Ortega S, CarralónGonzález MM, et al. Clinical characteristics and outcomes among hospitalized adults with severe COVID-19 admitted to a tertiary medical center and receiving antiviral, antimalarials, glucocorticoids, or immunomodulation with tocilizumab or cyclosporine: a retrospective observational study (COQUIMA cohort). EClinicalMedicine. 2020;28: 100591.

47. Klopfenstein $\mathrm{T}$, Zayet $\mathrm{S}$, Lohse $\mathrm{A}$, et al. Impact of tocilizumab on mortality and/or invasive mechanical ventilation requirement in a cohort of 206 COVID-19 patients. Int J Infect Dis. 2020;99:491-5.

48. Rossi B, Nguyen LS, Zimmermann P, et al. Effect of tocilizumab in hospitalized patients with severe COVID-19 pneumonia: a case-control cohort study. Pharmaceuticals (Basel). 2020;13:317.

49. Rosas IO, Bräu N, Waters M, et al. Tocilizumab in hospitalized patients with COVID-19 pneumonia. medRxiv. 2020:2020.08.27.20183442.

50. Veiga VC, Prats J, Farias DLC, et al. Effect of tocilizumab on clinical outcomes at 15 days in patients with severe or critical coronavirus disease 2019: randomised controlled trial. BMJ. 2021;372:n84.

51. Kumar S, de Souza R, Nadkar M, et al. A two-arm, randomized, controlled, multi-centric, open-label Phase-2 study to evaluate the efficacy and safety of Itolizumab in moderate to severe ARDS patients due to COVID-19. medRxiv. 2020:2020.12.01. 20239574.

52. Horby PW, Pessoa-Amorim G, Peto L, et al. Tocilizumab in patients admitted to hospital with COVID-19 (RECOVERY): preliminary results of a randomised, controlled, open-label, platform trial. medRxiv. 2021:2021.02.11.21249258.

53. Soin AS, Kumar K, Choudhary NS, et al. Tocilizumab plus standard care versus standard care in patients in India with moderate to severe COVID19-associated cytokine release syndrome (COVINTOC): an open-label, multicentre, randomised, controlled, phase 3 trial. Lancet Respir Med. 2021;9: 511-21.

54. Gordon AC, Mouncey PR, Al-Beidh F, et al. Interleukin-6 receptor antagonists in critically ill patients with covid-19. N Engl J Med. 2021;384: 1491-502. 